Oper Orthop Traumatol 2020 · 32:475-476

https://doi.org/10.1007/s00064-020-00687-3

(c) Springer Medizin Verlag GmbH, ein Teil von Springer Nature 2020

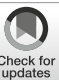

\author{
Frank Unglaub ${ }^{1,2} \cdot$ Michael Sauerbier $^{3}$ \\ 'Handchirurgie, Vulpiusklinik, Bad Rappenau, Deutschland \\ ${ }^{2}$ Medizinische Fakultät Mannheim, Universität Heidelberg, Heidelberg, Deutschland \\ ${ }^{3}$ PROFESSOR SAUERBIER, Privatärztliche Praxis für Hand- und Plastische Chirurgie, Bad Homburg v. d. \\ Höhe, Deutschland
}

\title{
Defektdeckung an der Hand
}

Liebe Leserinnen und Leser,

aufgrund der Exposition der Hände im Alltag sind Weichteildefekte in dieser Region eine der häufigsten Verletzungen überhaupt. Die Schwere der Verletzung reicht von marginalen Fingerkuppendefekten bis hin zu umfangreichen Hautweichteilverlusten mit freiliegenden Sehnen, Nerven und Gefäßen. Häufig ist das genaue Ausmaß der Verletzung erst nach einem sorgfältigen Débridement einzuschätzen und dann auch erst die Entscheidung der Defektdeckungsvariante möglich. Die eigentliche Herausforderung besteht darin, das sinnvollste Rekonstruktionsverfahren zu wählen, welches mit dem kleinstmöglichen Hebedefekt einhergeht und eine gute postoperative Funktion der Hand ermöglicht. Auch das ästhetische Erscheinungsbild sollte in diese Entscheidung mit „einfließen“.

Trotz der zunehmenden „Attraktivität“ des Semiokklusionsverbandes [1] ist ein angemessenes Repertoire an Lappenplastiken für den Handchirurgen notwendig zur Versorgung ausgedehnterer Verletzungen. In den letzten Jahren wurden in Operative Orthopädie und Traumatologie verschiedene, etablierte Defektdeckungsverfahren an der Hand veröffentlicht, die weiter unten aufgelistet sind. Erwähnt sind auch Lappenplastiken, die häufig bei elektiven Eingriffen Anwendung finden, wie beispielsweise bei der operativen Therapie des Morbus Dupuytren [2]. Diese Operationstechniken sind teilweise auf traumatische Defektverletzungen übertragbar und können so das Verständnis und das eigene Repertoire für lokale Lappenplastiken an der Hand erweitern.
Dieses Schwerpunktheft soll nun die Liste der bereits veröffentlichten Lappenplastiken durch weitere, etablierte Techniken ergänzen. Alle Manuskripte wurden von erfahrenen Handchirurgen/Plastischen Chirurgen angefertigt und geben sinnvolle Tipps und Tricks, um eine erfolgreiche Wiederherstellung von Funktion und Form der Hand zu ermöglichen.

Wir würden uns freuen, wenn unsere Leserinnen und Leser von diesem Schwerpunktheft für ihre Patientenversorgung profitieren können.

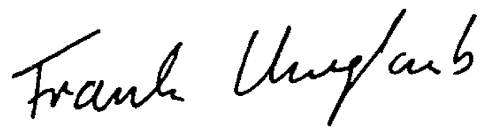

Prof. Dr. med. Frank Unglaub

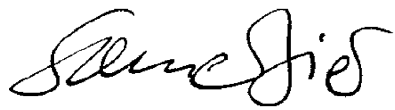

Prof. Dr. med. Dr. med. habil. Michael Sauerbier

\section{Korrespondenzadresse

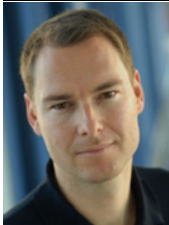 \\ Prof. Dr. med. Frank Unglaub Handchirurgie, Vulpiusklinik Vulpiusstr. 29, 74906 Bad Rappenau, Deutschland unglaub@vulpiusklinik.de

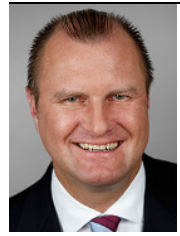 \\ Prof. Dr. med. Dr. med. habil. Michael Sauerbier PROFESSOR SAUERBIER, Privatärztliche Praxis für Hand- und Plastische Chirurgie Louisenstr. 92, $61348 \mathrm{Bad}$ Homburg v. d. Höhe, Deutschland sauerbier@profsauerbier.com}

Beiträge zum Thema Lappenplastiken an der Hand" 


\section{Einführung zum Thema}

Interessenkonflikt. F. Unglaub und M. Sauerbier geben an, dass kein Interessenkonflikt besteht.

\section{Literatur}

\section{Verwendete Literatur}

1. Mühldorfer-Fodor $M$, Hohendorff $B$, Vorderwinkler KP, van Schoonhoven J, Prommersberger KJ (2013) Behandlung von Fingerkuppendefektverletzungen mit dem Semiokklusionsverband nach Mennen und Wiese. Oper Orthop Traumatol 25(1):104-114

2. Hahn P, Unglaub F (2016) Operative Therapie bei Morbus Dupuytren. Oper Orthop Traumatol 28(1):2-3

\section{Weiterführende Literatur}

3. Lukas B, Lukas M (2016) Lappenplastiken bei fortgeschrittenem Morbus Dupuytren. Oper Orthop Traumatol 28(1):20-29

4. Walle L, Hohendorff $B$, Pillukat T, van Schoonhoven J (2016) Laterodorsaler Transpositionslappen zum Verschluss eines Weichteildefekts am palmaren Kleinfingergrundglied nach Auflösung einer Dupuytren-Rezidivbeugekontraktur. Oper Orthop Traumatol 28(1):38-45

5. Stang F, Siemers F, von Wild T, Weyers I, Mailänder P, Stollwerck P (2013) Die M.-abductor-digitiminimiLappen plastikzurDefektdeckung im Handbereich. Oper Orthop Traumatol 25(4):372-380

6. Harenberg PS, Jakubietz RG, Jakubietz MG, Schmidt K, Meffert RH (2012) Reconstruction of the thumb tip using palmar neurovascular flaps. Oper Orthop Traumatol 24(2):116-121

7. Saalabian A, Rab M, van Schoonhoven J, Prommersberger KJ (2009) Insellappenplastikderersten dorsalen Mittelhandarterie nach Foucher. Oper Orthop Traumatol 21(6):614-619

8. Stang F, Rab M, van Schoonhoven J, Prommersberger KJ (2008) Der dorsale Fähnchenlappen zur Defektdeckung im Finger- und Daumenbereich. Oper Orthop Traumatol 20(3):221-227

9. Rab M, Prommersberger KJ (2008) Die distal gestielte Arteria-interossea-posterior-Lappenplastik zur Defektdeckung am Handgelenk und an der Hand. Oper Orthop Traumatol 20(2):111-118

10. Megerle K, Palm-Bröking K, Germann G (2008) Der Crossfingerlappen. Oper Orthop Traumatol 20(2):97-102

11. Voigt M, Schaefer DJ, Stark GB (1999) Der Interosseuslappen bei Weichteildefekten an Unterarm und Hand. Oper Orthop Traumatol 11(3):183-196

\section{Aktuelle Entwicklung zu COVID-19}

Auf der Homepage SpringerMedizin.de finden Sie ein immer aktuelles Dossier mit Beiträgen, Forschungsarbeiten und Ergebnissen zu SARS-CoV-2 sowie relevanten Links.

SpringerNature.com/de verlinkt auf eine eigene Landingpage mit einer Vielzahl an Information sowie freiem Zugriff auf die COVID-19-Contentplatformen von Nature Research, BioMed Central (BMC) und Springer. 